\title{
Genetic relationships among twelve Chinese indigenous goat populations based on microsatellite analysis
}

\author{
Meng-Hua $\mathrm{Li}^{\mathrm{a}}$, Shu-Hong $\mathrm{ZHAO}^{\mathrm{a} *}$, \\ Ci BIAN ${ }^{b}$, Hai-Sheng WANG ${ }^{\mathrm{a}, \mathrm{c}}$, Hong WeI ${ }^{\mathrm{d}}$, Bang LiU ${ }^{\mathrm{a}}$, Mei Yu ${ }^{\mathrm{a}}$, \\ Bin FAN ${ }^{\mathrm{a}}$, Shi-Lin CHEN ${ }^{\mathrm{a}}$, Meng-Jin ZHU ${ }^{\mathrm{a}}$, Shi-Jun Li ${ }^{\mathrm{a}}$, \\ Tong-An XIONG ${ }^{\mathrm{a}}$, Kui Li ${ }^{\mathrm{a}}$ \\ ${ }^{a}$ Laboratory of Molecular Biology and Animal Breeding, \\ School of Animal Husbandry and Veterinary Medicine, \\ Huazhong Agricultural University, Wuhan 430070, P.R. China \\ ${ }^{b}$ Department of Animal Science, Tibet Agriculture and Animal Husbandry College, \\ Linzhi 860000, P.R. China \\ ${ }^{\mathrm{c}}$ Institute of Criminal Science and Technology, \\ The Public Security Bureau of Hubei Province, Wuhan 430070, P.R. China \\ ${ }^{\mathrm{d}}$ Laboratory Animal Center, Third Military Medical University, \\ Chongqin 400038, P.R. China
}

(Received 26 October 2001; accepted 4 June 2002)

\begin{abstract}
Twelve Chinese indigenous goat populations were genotyped for twenty-six microsatellite markers recommended by the EU Sheep and Goat Biodiversity Project. A total of 452 goats were tested. Seventeen of the 26 microsatellite markers used in this analysis had four or more alleles. The mean expected heterozygosity and the mean observed heterozygosity for the population varied from 0.611 to 0.784 and 0.602 to 0.783 respectively. The mean $F_{\mathrm{ST}}$ (0.105) demonstrated that about $89.5 \%$ of the total genetic variation was due to the genetic differentiation within each population. A phylogenetic tree based on the Nei (1978) standard genetic distance displayed a remarkable degree of consistency with their different geographical origins and their presumed migration throughout China. The correspondence analysis did not only distinguish population groups, but also confirmed the above results, classifying the important populations contributing to diversity. Additionally, some specific alleles were shown to be important in the construction of the population structure. The study analyzed the recent origins of these populations and contributed to the knowledge and genetic characterization of Chinese indigenous goat populations. In addition, the seventeen microsatellites recommended by the EU Sheep and Goat Biodiversity Project proved to be useful for the biodiversity studies in goat breeds.
\end{abstract}

\section{genetic relationship / microsatellite / goat / Chinese indigenous population}

* Correspondence and reprints

E-mail: shzhao@mail.hzau.edu.cn 


\section{INTRODUCTION}

Goats were first domesticated in west Asia during the period of 90007000 B.C. [35]. They migrated east into China. Modern goat breeds generally originated from the territorial plateau of southwest China and the adjacent mid-Asia area [28]. There are 135.92 million goats in China [18] and the Chinese indigenous goat breeds are a valuable resource in the world goat population. Twelve Chinese indigenous goat populations were investigated in this study: Tibetan goats distributed among the Qinhai-Tibet Plateau. The Tibetan goats, having a strong adaptability prefer the cold weather over the dry climate. The Tibetan goats are divided into two ecotypes according to their ecological characteristics such as body figure, fur, dissection, physiological and biochemical indices: the plateau one and the mountain-valley one [30]. The Wu goat, Nanjiang Brown goat, Black goat and Chuandong white goat exist in the isolated Three-gorge reservoir area. The $W u$ goat, also named the "medical goat", provided a medical value. The Small-xiang goat originates from the remote mountain area of the Guizhou province in southwest China. In order to maintain its small physical figure and fragrance after being cooked, intercrosses are often made and the population size of the small-xiang goat has become smaller. Three breeds (Neimonggol, Liaoning, Taihang) originating from north China and one local breed from central China are famous for cashmere, down, and mutton respectively.

The evolution of goat breeds has been shaped by man over many generations. The local climates, diseases, nutritional environments, selections for different objectives and genetic drifts have contributed to the evolution of diverse goat breeds. As a result of the introduction of modern commercial goat breeds and the shortage of effective conservation, some populations, such as the $W u$ goat, Small-xiang goat and Tibetan goat, have decreased rapidly in number of sires and population sizes. Some are even facing extinction. Since the genetic resources required for the future are difficult to predict, selection for conserving these populations with unique evolutionary history has to be taken into account and breeds should be chosen in order to cover the widest range of genetic variability. In addition, the Three-gorge Project will force some goat populations to leave the habitat they have occupied for centuries. Therefore, the evaluation of the genetic structure, conservation and utilization of these goat breeds are urgent tasks for animal breeders and geneticists.

In recent years, the genetic diversity of Chinese indigenous goat breeds has been evaluated on the basis of biochemical genetic methods [30], mitochondrial DNA (mt DNA) restriction patterns [15] and random amplified polymorphic DNA (RAPD) [8]. However, all of these markers are polymorphic, but not highly variable and serum proteins have not revealed a clear separation of the plateau type and the mountain-valley type of Tibetan goats [31]. Microsatellite 


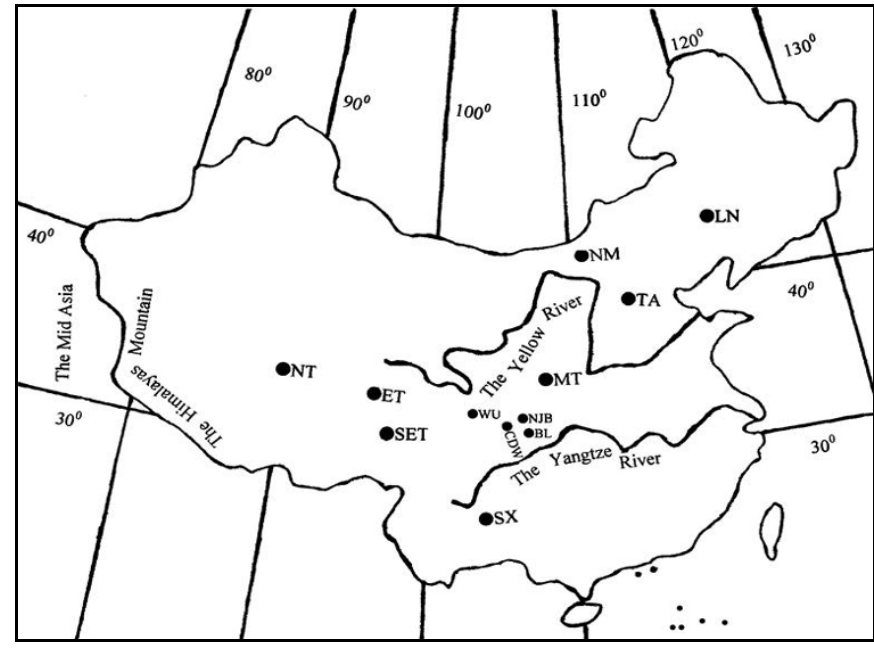

Figure 1. Geographical locations of the 12 Chinese indigenous goat populations sampled. The two-letter or three-code letter besides the black point in the figure corresponds to the populations sampled as follows: East Tibetan goat, ET; Neimonggol goat, NM; Liaoning goat, LN; Taihang goat, TA; Wu goat, WU; Nanjiang Brown goat, NJB; Chuandong White goat, CDW; Black goat, BL; Matou goat, MT; South-east Tibetan goat, SET; North Tibetan goat, NT; Small-xiang goat, SX.

DNA is currently the most useful marker of choice for a wide range of molecular genetic studies such as establishing population structure [5], population differentiation and reconstruction of phylogenetic relationships among populations $[4,16,32]$. The present study was undertaken to characterize the general relationships among twelve indigenous goat populations by estimating genetic distances from 17 microsatellites. This total includes two microsatellite loci screened across five goat populations previously studied in this laboratory [33].

\section{MATERIALS AND METHODS}

\subsection{Sample collection for DNA analysis}

A total of 452 randomly sampled animals from different geographical locations representing twelve Chinese indigenous populations was analyzed. Southeast Tibetan goats, North Tibetan goats and East Tibetan goats were sampled in particular villages and towns of different ecological zones within the Tibet autonomous region and were grouped according to these ecological zones. Sample size and locality for each population are listed in Table I. The geographical distributions of these populations are shown in Figure 1. 


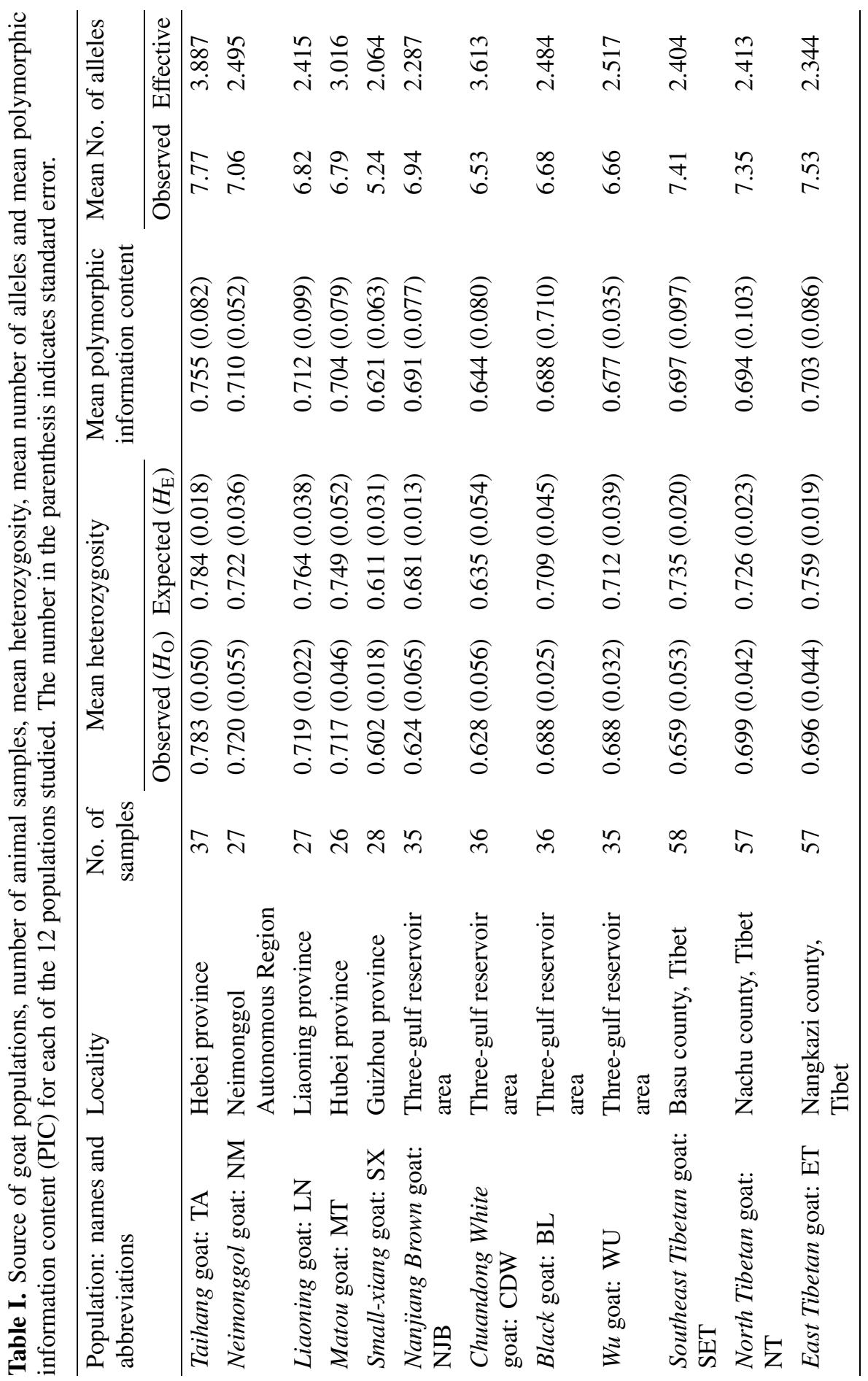




\subsection{DNA extraction and PCR amplification}

Blood collection and DNA extraction were conducted in accordance with Li et al. [14]. A total of 26 microsatellite markers recommended by the EU Sheep and Goat Biodiversity Project (http://139.222.64.94) were included in this investigation. The PCR amplification protocol was based on Crawford et al. [9]. Fluorescently end-labeled (with fluorescent dye: FAM, JOE; the internal size standard: Genescan-Rox500) PCR primers were used and size characterization of the PCR product was performed with an ABI 310 DNA Genetic Analyzer (Applied Biosystem/Perkin Elmer, Foster City, CA, USA).

\subsection{Data analysis}

\subsubsection{Diversity analysis}

The allele frequencies and tests of genotype frequencies for deviation from Hardy-Weinberg equilibrium (HWE) were carried out using the exact tests of the GENEPOP v.1.2 program [23]. The GENES IN POPULATIONS v.2.0 program [17] was employed for the calculation of total heterozygosity $\left(H_{\mathrm{T}}\right)$, expected heterozygosity $\left(H_{\mathrm{S}}\right)$ for locus, mean observed heterozygosity $\left(H_{\mathrm{O}}\right)$ and mean expected heterozygosity $\left(H_{\mathrm{E}}\right)$ for populations. The Wright FStatistic for locus, polymorphic information content (PIC) [3] and effective allele number [11] were calculated using the $\mathrm{SAS}^{\circledR}$ software package [24].

The standard genetic distance of Nei (1978) [19] and Cavalli-Sforza and Edwards (1967) [6] chord distance, calculated from the allele frequencies, demonstrated their superior performance in phylogenetic tree construction when the microsatellite marker was used [27]. For the purpose of comparing our results with those obtained by other authors [29,34], Nei (1978) standard genetic distances were estimated using the DISPAN package [21]. The genetic affinities among the twelve analyzed populations were evaluated by the neighbor-joining tree. Bootstrap $(n=1000)$ resampling was performed to test the robustness of the dendrogram topology.

\subsubsection{Multivariate correspondence analysis}

Multivariate analysis deals with the statistical analysis of the data collected on more than one variable and can condense the information from a large number of alleles and loci into fewer synthetic variables. Correspondence analysis (CA) $[2,13]$ is a multivariate method analogous to the principal component analysis (PCA) but which is appropriate for discrete variables. It is applied to study the link between and to seek the best simultaneous representation of two sets of categories that make up the rows and columns of a contingency table, where these two sets have symmetrical roles. Correspondence analysis (CA) can also be transformed into principal component analysis (PCA) by 
making appropriate changes to variables. A correspondence analysis (CA) was performed to reveal major patterns of genetic variability based on the allele frequencies among all the populations.

\section{RESULTS}

\subsection{Genetic variability}

Allele frequencies are available from the authors upon request. All the Chinese indigenous goat populations were genetically highly diverse at 17 loci of the total 26 loci (Tab. II). Specific alleles were present in some populations. The breed-specific allele of $B M 2113$ (157 bp) was present with a frequency of $74 \%$ only in the three populations of the Southeast Tibetan goat, North Tibetan goat and East Tibetan goat. The unique alleles of MAF70 (142 bp) and SR-CRSP-1 (138 bp) were found only in the Matou goat and Small-xiang goat respectively. Among the 26 loci, 17 were polymorphic and the number of alleles varied between 4 (ILSTSO05) and 19 (BM2113). The remaining nine loci tested had less than four alleles or non-specific PCR products. It was suggested by Barker [1] that, for studies of genetic distance, microsatellite loci should have no fewer than four alleles to reduce the standard errors of distance estimates; so nine loci were excluded from this analysis. Mean observed heterozygosities, mean expected heterozygosities, mean polymorphic information content (PIC) and their standard errors respectively, mean observed number of alleles, and mean effective number of alleles for all populations are shown in Table I. Although varying among populations, observed mean heterozygosity was lower than the expected mean heterozygosity for all the populations. Measures of genetic variation for each population showed that the level of genetic variation within the Taihang goat population was the highest and that of the Small-xiang goat was the lowest.

The $H_{\mathrm{T}}, H_{\mathrm{S}}$, fixation indices ( $F_{\mathrm{IS}}, F_{\mathrm{IT}}$ and $F_{\mathrm{ST}}$ ) values for each locus are shown in Table II. The $H_{\mathrm{T}}$ varied from 0.657 (ILSTSO05) to 0.880 (BM2113). Multilocus $F_{\mathrm{ST}}$ values indicated that around $10.5 \%$ of the total genetic variation was explained by a population difference, the remaining $89.5 \%$ corresponding to differences among individuals. The HWE test showed that all loci gave a deviation from the HWE when analyzed across populations. On the contrary, the three Tibetan goat populations were in equilibrium for all 17 loci when pooled across loci. By contrast, the mean observed numbers of alleles and the mean expected heterozygosities in the three populations of the Tibetan goat breed were higher than the majority of the nine other populations (eight and six respectively). The main factors that may have caused such deviations in the remaining populations are probably their small effective population sizes and the difficulties in collecting enough unrelated pure individuals. 
Genetic relationships among goats

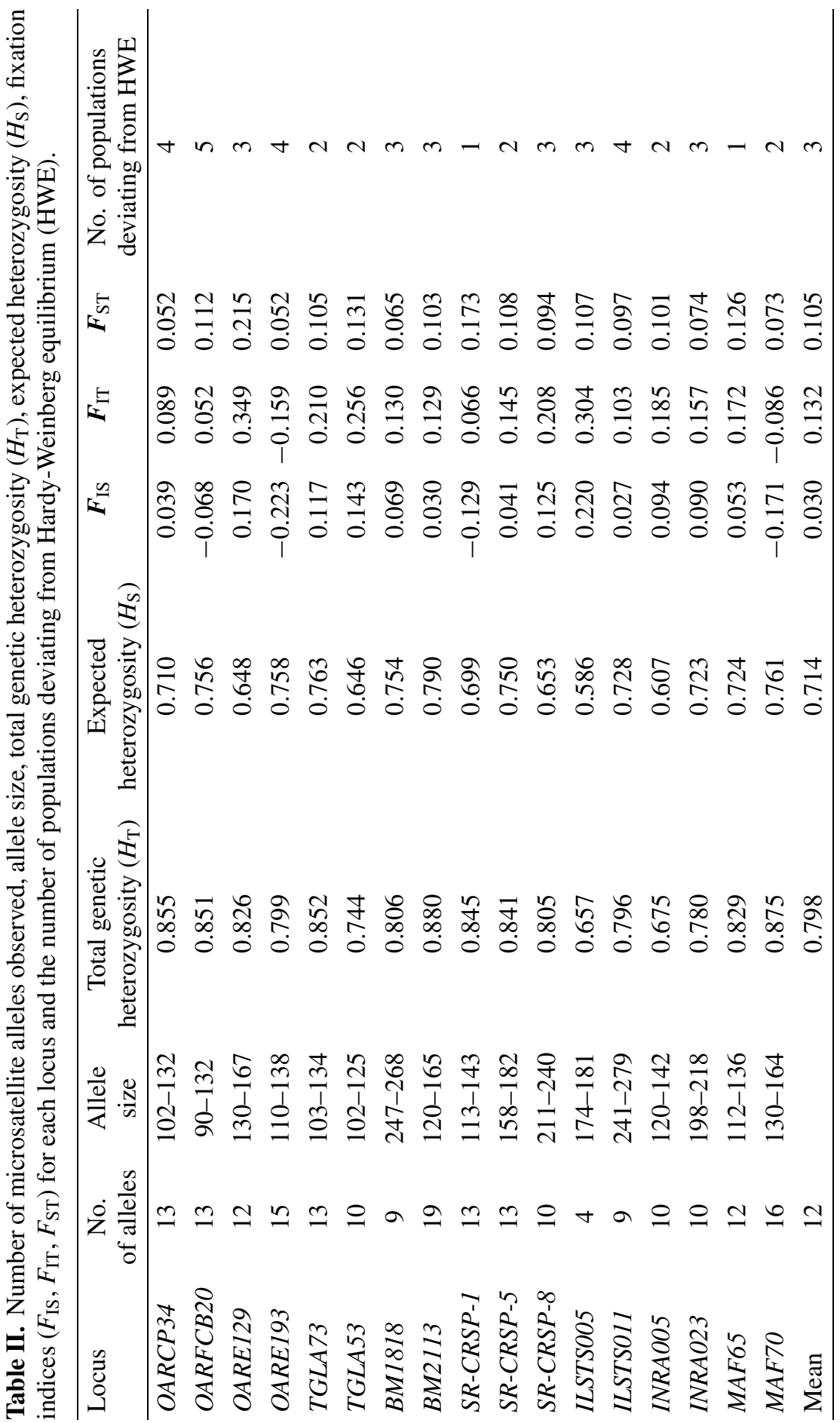


Table III. Matrix of genetic distance among 12 goat populations: the Nei [1978] standard genetic distances (below diagonal) and standard errors (above diagonal).

\begin{tabular}{lcccccccccccc}
\hline & ET $^{(1)}$ & NM & LN & TA & WU & NJB & CDW & BL & MT & SET & NT & SX \\
\hline ET & & 0.073 & 0.078 & 0.097 & 0.087 & 0.045 & 0.047 & 0.073 & 0.095 & 0.083 & 0.087 & 0.069 \\
NM & 0.176 & & 0.052 & 0.086 & 0.057 & 0.040 & 0.042 & 0.055 & 0.036 & 0.068 & 0.079 & 0.062 \\
LN & 0.259 & 0.230 & & 0.062 & 0.084 & 0.063 & 0.045 & 0.089 & 0.089 & 0.078 & 0.039 & 0.060 \\
TA & 0.419 & 0.315 & 0.513 & & 0.077 & 0.075 & 0.061 & 0.035 & 0.094 & 0.024 & 0.056 & 0.043 \\
WU & 0.291 & 0.255 & 0.386 & 0.354 & & 0.081 & 0.047 & 0.053 & 0.022 & 0.075 & 0.022 & 0.100 \\
NJB & 0.205 & 0.227 & 0.289 & 0.472 & 0.331 & & 0.064 & 0.080 & 0.069 & 0.042 & 0.039 & 0.038 \\
CDW & 0.478 & 0.409 & 0.545 & 0.396 & 0.412 & 0.433 & & 0.040 & 0.047 & 0.073 & 0.062 & 0.030 \\
BL & 0.324 & 0.318 & 0.516 & 0.183 & 0.183 & 0.386 & 0.304 & & 0.049 & 0.097 & 0.083 & 0.099 \\
MT & 0.290 & 0.195 & 0.423 & 0.236 & 0.073 & 0.300 & 0.303 & 0.128 & & 0.057 & 0.024 & 0.088 \\
SET & 0.379 & 0.316 & 0.376 & 0.490 & 0.336 & 0.206 & 0.465 & 0.447 & 0.343 & & 0.054 & 0.060 \\
NT & 0.324 & 0.375 & 0.441 & 0.525 & 0.427 & 0.193 & 0.271 & 0.440 & 0.394 & 0.249 & & 0.081 \\
SX & 0.320 & 0.335 & 0.294 & 0.514 & 0.423 & 0.236 & 0.559 & 0.421 & 0.408 & 0.299 & 0.256 & \\
\hline
\end{tabular}

(1) The two-letter and three-code letter in the table correspond to the populations sampled as follows: East Tibetan goat, ET; Neimonggol goat, NM; Liaoning goat, LN; Taihang goat, TA; Wu goat, WU; Nanjiang Brown goat, NJB; Chuandong White goat, CDW; Black goat, BL; Matou goat, MT; South-east Tibetan goat, SET; North Tibetan goat, NT; Small-xiang goat, SX.

\subsection{Genetic distances}

In the Chinese indigenous goat groups, genetic differentiation was significant between the populations originating in different ecological zones. Among the Tibetan goat populations, a close relationship was shown between the genetic distances determined for the North Tibetan and the East Tibetan goat populations (Tab. III). A NJ topology tree based on the Nei (1978) standard genetic distance relating the 12 indigenous goat populations studied is presented in Figure 2. The numbers at the nodes are values for 1000 bootstrap resampling of the 17 typed loci. The bootstrap values obtained in the NJ topology tree at the nodes suggest that the robustness of the tree is not high, but the genetic relationships of the Chinese indigenous goat populations fit well with their geographic origins from the $\mathrm{NJ}$ topology tree.

\subsection{Correspondence analysis}

Figure 3 illustrates the three-factor correspondence analysis for 17 microsatellite allele frequency distributions in 12 Chinese indigenous goat populations. The first two factors accounted for $28 \%$ and $18 \%$ of the total variation respectively and clearly distinguished the following blocks: block I (South-east 


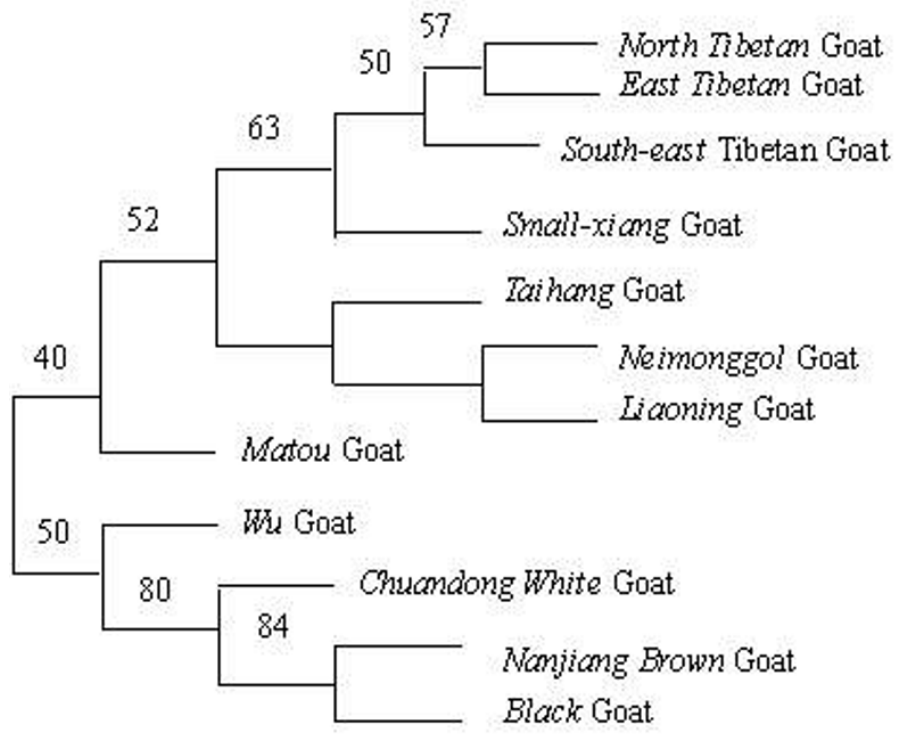

Figure 2. The NJ topology tree showing the genetic relationships among goat populations using the Nei [1978] standard genetic distance from 17 microsatellite loci. The numbers at the nodes indicate the percentage of a group's occurrence in a bootstrap resampling of 1000 trees.

Tibetan goat, North Tibetan goat, East Tibetan goat, Small-xiang goat), block II (Taihang goat, Neimonggol goat, Liaoning goat) and block III (Nanjiang Brown goat, Chuandong White goat, Black goat, Wu goat). The Matou goat population was isolated from the others and represented $12 \%$ of the total variation respective to the other populations. The first two dimensions fitted well with the geography, while the third factor, contributing $14 \%$ of the total variation, played an important role in discriminating the Small-xiang goat population.

The most important alleles are allele $B M 2113$ (157 bp) which contributed $12 \%$ in axis 1 and $8 \%$ in axis 2, allele MAF70 (142 bp) which contributed 9\% in axis 1 and $14 \%$ in axis 2 and allele $S R-C R S P-1$ (138 bp) which contributed $9 \%$ in axis 2 and $15 \%$ in axis 3 . The $B M 2113$ allele (157 bp) is a breed-specific allele with frequencies of $38 \%, 42 \%$ and $32 \%$ in the South-east Tibetan goat population, North Tibetan goat population and East Tibetan goat population respectively. The unique alleles of allele $\operatorname{MAF70}(142 \mathrm{bp})$ and allele $S R$ CRSP-1 (138 bp) which were closely associated with the Matou goat breed and Small-xiang goat breed, respectively, were present with frequencies of $42 \%$ in the Matou goat population and $49 \%$ in the Small-xiang goat population. Considering the important effect of the three breed-specific alleles, we repeated the analysis excluding the three microsatellites separately. As a result, the Small-xiang goat went into the cluster of the South-east Tibetan goat, North 
(A)

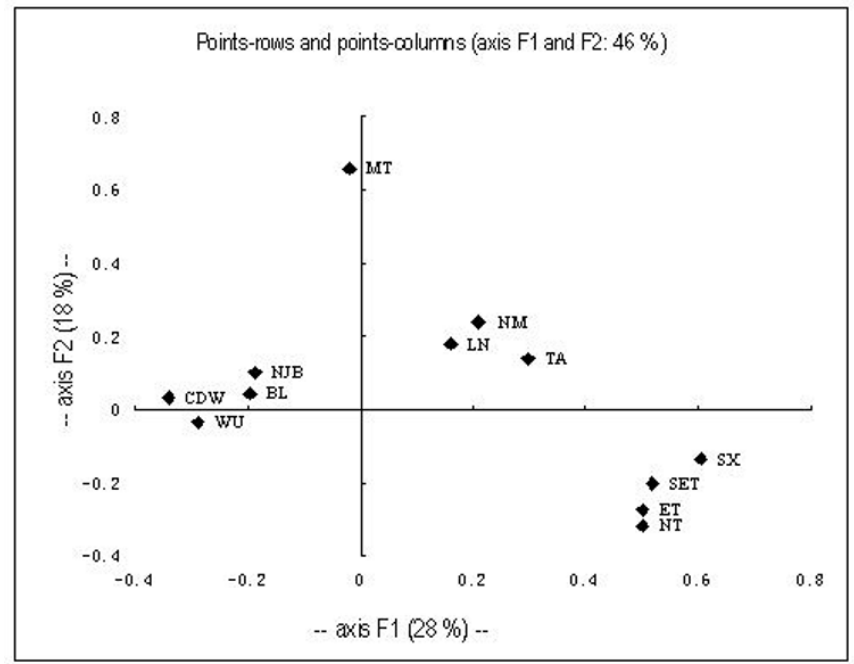

(B)

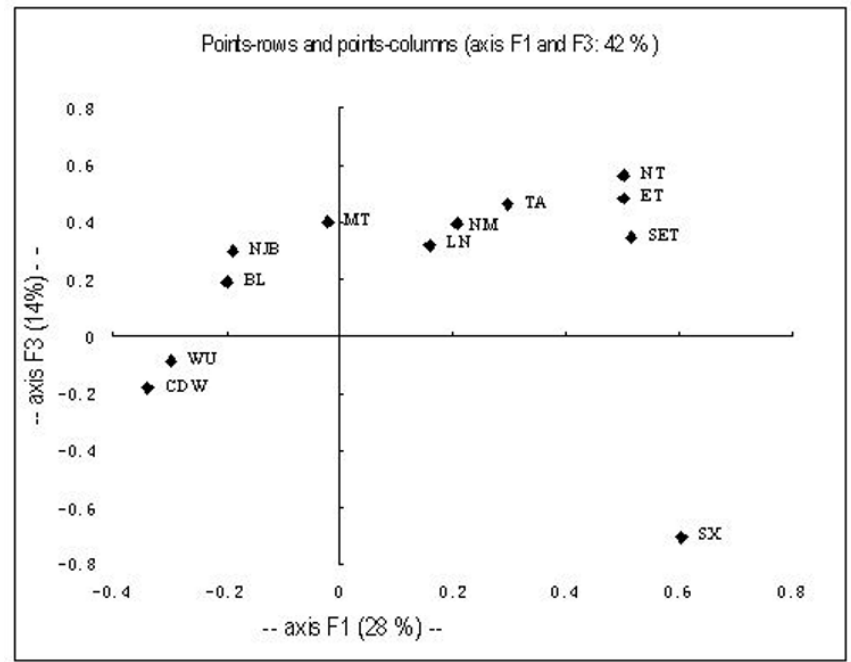

Figure 3. Correspondence analysis of allele frequencies from seventeen microsatellite loci genotyped in twelve Chinese indigenous goat populations: (A) projection of populations on axis 1 and axis 2; (B) projection of populations on axis 1 and axis 3 . The two-letter and three-code letter in the figure correspond to the populations sampled as follows: East Tibetan goat, ET; Neimonggol goat, NM; Liaoning goat, LN; Taihang goat, TA; Wu goat, WU; Nanjiang Brown goat, NJB; Chuandong White goat, CDW; Black goat, BL; Matou goat, MT; South-east Tibetan goat, SET; North Tibetan goat, NT; Small-xiang goat, SX. 
Tibetan goat and East Tibetan goat for excluding the microsatellite $S R-C R$ $S P-1$ and the Matou goat went into the cluster of the Nanjiang Brown goat, Chuandong White goat, Black goat and $W u$ goat for removing the MAF70 microsatellite. Some separation still existed between the cluster of the Southeast Tibetan goat, North Tibetan goat, East Tibetan goat and the rest of the populations after removing BM2113 microsatellite from the analysis, though the result was less robust than before. On the contrary, when we repeated the analysis excluding one by one the breeds in which there was a breed-specific allele, a zooming-in effect on the other populations appeared in the results. These two changes were also reported by Cañón J. et al. [10].

\section{DISCUSSION}

\subsection{Genetic variability within populations}

Heterozygosity estimates within the populations were based on a set of microsatellites showing that the Taihang goat had the largest genetic variability, whereas the Small-xiang goat showed the lowest genetic variability. The mean number of alleles and mean observed and expected heterozygosities were similar (Tab. I), supported by $F_{\text {IS }}$ estimates that were not significantly different from zero (Tab. II). The cause may be that the Taihang goat had a large number of individuals and broad distributing area. In contrast, the Small-xiang goat existed in a remote area with a small population size and there was less gene exchange between it and other populations. However, it is well known that the number of alleles in a population is a function of sample size. In a population, larger sample size would result in more alleles. To reduce the impact of population size on comparing the mean numbers of alleles between populations, resampling under a constant size should be an effective alternative. The mean observed heterozygosity and mean polymorphic information content (PIC) of the three Tibetan goat populations were lower than those of the Taihang, Matou and Neimonggol goat populations. This result was in concordance with that of six microsatellite loci reported previously by Yang et al. [33]. Comparisons of the mean observed heterozygosity, mean polymorphic information content (PIC) and mean observed number of alleles between the four goat populations originating in the Three-gorge reservoir area and the other goat populations except the Small-xiang goat indicate that the polymorphisms of the four goat populations from the Three-gorge reservoir area are slightly lower. A possible explanation for this observation may be that the rapidly reduced population size and the isolated geographic location resulted from the Three-gorge Project.

Intercrosses with other goat populations may result in that the $W u$ goat is more polymorphic than the three other goat populations existing in the Threegorge reservoir area. The mean observed heterozygosity over all populations 
is higher than that of eight Swiss goat breeds, the Creole goatand Bezoar goat [25]. Since the set of microsatellites we used showed a little higher variability than that of the microsatellites used in the genetic diversity analysis of Swiss goat breeds, the Creole goat and Bezoar goat, we interpreted our higher gene values as reflections of both the choice of the microsatellite and the choice of populations. The existence of null alleles has been frequently reported, particularly when the markers are transferred between species. In this study, the clear deficiency of polymorphism at the other nine loci in all Chinese indigenous breeds suggests that they are not promising for studies on genetic diversity analysis of goat breeds. In the global test of deviation from Hardy-Weinberg equilibrium, a number of locus-population combinations showed a significant departure (Tab. II). The deviations from the expected value may be due to a variety of causes: population subdivision owing to genetic drift [12] or the effect of a bottleneck through the reproductive isolation of rare populations [27], whereas the equilibrium in the three populations of the Tibetan goat for all loci may result from a large effective population size, their few artificial selections and random mating in the populations.

\subsection{Genetic variability between populations}

Genetic relationships among the populations are illustrated by the NJ topology tree derived from the Nei (1978) standard genetic distance. Although the NJ topology tree is not well supported by the nodes, the dendrogram (Fig. 2) shows a clear separation of the Chinese indigenous goat populations from different geographic locations. Since some goat populations may be derived from a small number of founders, possible bottleneck effects should not be ignored in interpreting the population relationships [20]. The mean $F_{\mathrm{ST}}$ value $(0.105)$ demonstrates that only about $10.5 \%$ of the total genetic variation attributes to the differentiation between populations and $89.5 \%$ is within the populations. This result is lower than that of the total populations including eight Swiss goat breeds, the Creole goat and Bezoar goat (0.27) [25]. Among the Chinese indigenous goats, breeds are mainly artefacts classically based on morphological differences and tightly related to geographical locations. Within the tree, three sub-clusters can be identified which contain the populations from southwest China, north China and the Three-gorge reservoir area. The Matou goat originating in central China at some distance from the other Chinese indigenous populations forms a sub-branch alone, which has been reported previously, based on the analysis of blood group [26] and six microsatellite loci [34].

In the subgroup of the Three-gorge reservoir area, the NanJiang Brown goat was closely grouped with the Black goat. This was consistent with the recorded breed history and the result of a random amplified polymorphic DNA (RAPD) molecular marker [33]. The Nanjiang Brown goat was formed by crossing 
between the Black goat and Chengdu Grey goat. Moreover, the Black goat usually was considered as a type of the Chuandong White goat. The Wu goat had a common geographical location and a similar morphological appearance to that of the Chuandong White goat. In general, the four populations had closer genetic distances and relationships.

There are three populations in the sub-cluster of north China. The Taihang goat separates itself from the Liaoning goat and Neimonggol goat for fleece characters since it is assumed that such a difference reflects distinct origins. The three populations studied in this paper are the Liaoning goat and Neimonggol goat (coarl-wool type), and the Taihang goat (fine-wool type). The four populations from southwest China form a subgroup. Reproductive isolation by geographic barriers led to the genetic differentiation between the Small-xiang goat and the three other Tibetan goat populations. Among the three Tibetan goat populations, the dendrogram showed a separation of the plateau type (North Tibetan goat, East Tibetan goat) and mountain-valley type (South-east Tibetan goat). This was in concordance with the non-negligible difference between the two types of the Tibetan goats not obtained in some previous studies using random amplified polymorphic DNA (RAPD) and restriction fragment length polymorphism (RFLP) markers [22].

Concerning the correspondence analysis, our findings were in perfect agreement with the historic and geographic origins of the twelve Chinese indigenous goat populations. From Figure 3 it is evident that axis 1 has an important effect on the genetic differentiation of all the populations. Resulting from the presence of breed-specific alleles, the Matou goat and Small-xiang goat demonstrated separations from the other populations in Figure 3(A) and Figure 3(B) respectively. A distinct separation was the block of the Southeast Tibetan goat, North Tibetan goat and East Tibetan goat. Even though the populations of Taihang goat, Neimonggol goat and Liaoning goat were not very close to one another, the block was easily distinguished as well. Finally, there is the block of the Nanjiang Brown goat, Chuandong White goat, Black goat and $W u$ goat, although it was less homogeneous than the two blocks cited above. In this study, comparisons of the correspondence analysis with the neighbor-joining topology tree showed good agreement with each other. In addition, the results of the corresponding analysis excluding the three microsatellites separately indicated that the new population structures of the twelve goat populations were consistent with their geographic origins as well, although the new population structures were less precise than before.

The overall relationship pattern among the twelve Chinese indigenous goat populations proved that the middle valley of the Yellow River was the dissemination center of domestic goats in China. The blood lineage of the ancestor colonies in this area came from the Qinhai-Tibet plateau. The goats in this area spread eastwards and southwards after long periods of tameness [7]. The 
correspondence analysis (CA) was also in support of the results of the cluster analysis.

The results of this study contribute to the knowledge of the genetic structure of the Chinese indigenous goat populations, especially many of the small populations verging on the potential threat of extinction or even being effectively lost with the rapid destruction of the ecological environment. Conservation of genetic diversity should be considered by breeders, in the interest of the longterm future of the Chinese breeds. In addition, we conclude that the 17 loci of the microsatellite panel designed by the EU Sheep and Goat Biodiversity Project are suitable for the biodiversity studies in goats, even in closely related goat populations.

\section{ACKNOWLEDGEMENTS}

The authors would like to thank Professor Li Mingjia for the collection of goat blood samples, Professor Yu Jialin at the Huazhong Agricultural University, Dr. Jiang Xinping at the Yangzhou University and Zhu Chuanhong at the Institute of Criminal Science and Technology in Public Security Bureau of Wuhan City for part of the statistical analysis work. The authors would also like to thank the editor and the two anonymous referees for their good advice for the paper. In addition, we would like to thank Matthew Abbott and Terri Besch at the Iowa State University for revising the English language. The work was supported by the IFS (International Foundation for Science) Project (B/2580-2) to Dr. Shu-Hong Zhao, the regional foundation of National Natural Science Foundation of China (39960050) to Bian Ci and Dr. Shu-Hong Zhao, the National Outstanding Youth Science Foundation of China (39925027) and the Key Project of National Basic Research and Development Plan of China (G2000016103) to Dr. Kui Li.

\section{REFERENCES}

[1] Barker J.S.F., A global protocol for determining genetic distances among domestic livestock breeds, in: Proceeding of the 5th World Congress on Genetics Applied to Livestock Production, 7-12 August 1994, Vol. 21, University of Guelph, Ontario, pp. 501-508.

[2] Benzécri J.P., Analyse des données. Tome 2: Analyse des correspondances, Dunod, Paris, 1973.

[3] Bostein D., White R.L., Skolnick M., Davis R.W., Construction of a genetic linkage map in man using restriction fragment length polymorphism, Am. J. Hum. Genet. 32 (1980) 314-331.

[4] Bowcock A.M., Ruiz Linare A., Tomfohrde J., Minch E., Kidd J.R., CavalliSforza L.L., High resolution of human evolutionary trees with polymorphic microsatellites, Nature 368 (1994) 455-457. 
[5] Bruford M.W., Wayne R.K., Microsatellites and their application to population genetic studies, Curr. Opin. Genet. Dev. 39 (1993) 939-943.

[6] Cavalli-Sforza L., Edwards W., Phylogenetic analysis: models and estimation procedures, Evolution 21 (1967) 550-570.

[7] Chang H., Studies on Animal Genetic Resources in China, Shanxi People's Education Press, Xi' an (P.R. China), 1998.

[8] Chen S.L., Zhao S.H., Li Y.J., Li M.H., The research of random amplified polymorphic DNA(RAPD) of Liaoning cashmere goat, J. Huazhong Agr. Univ. 4 (2001) 303-305.

[9] Crawford A.M., Dodds K.G., Ede A.J., An autosomal genetic linkage map of the sheep genome, Genetics 140 (1995) 703-724.

[10] Cañón J., Alexandrino P., Bessa I., Carleos C., Carretero Y., Dunner S., Ferran N., Garcia D., Jordana J., Laloë D., Pereira A., Sanchez A., Moazami-Goudarzi K., Genetic diversity measures of local European beef cattle breeds for conservation purposes, Genet. Sel. Evol. 33 (2001) 311-332.

[11] Kimura M., Crow J.F., The number of alleles that can be maintained in a finite population, Genetics 49 (1964) 725-738.

[12] Lawson R., Kofron C.P., Dessauer H.C., Alloenzyme variation in natural populations of Nile crocodile, Am. Zool. 29 (1989) 863-871.

[13] Lebart L., Morineau A., Warwick K., Multivariate Descriptive Statistical Analysis, J. Wiley, New York, 1984.

[14] Li X., Gong Y., Zhao S., Li K., Peng Z., Isolation of DNA from the blood lysed in the pig farm, J. Hebei Agr. Univ. 20 (1997) 84-86.

[15] Li X., Zhang Y., Chen S., Zeng F., Study on the mtDNA RFLP of goat breeds, Zool. Res. 4 (1997) 421-428.

[16] MacHugh D.E., Loftus R.T., Cunningham P., Bradley D.G., Microsatellite DNA variation and the evolution, domestication and phylogeography of Taurine and Zebu cattle (Bos taurus and Bos indicus), Genetics 146 (1997) 1071-1086.

[17] May B., Krueger C.C., Eng C., Paul E., GENES IN POPULATIONS version 2.0: A Computer Program for Analysis of Genetic Data, Cornell Laboratory for Ecological and Evolutionary Genetics, Cornell University, New York, 1995.

[18] Ministry of Agriculture in People's Republic of China, The Chinese Agriculture Development Report, Beijing, 1996.

[19] Nei M., Estimation of average heterozygosity and genetic distance from a small number of individuals, Genetics 89 (1978) 583-590.

[20] Nei M., Maruyama T., Chakraborty R., The bottleneck effect and genetic variability in populations, Evolution 29 (1975) 1-10.

[21] Ota T., DISPAN: Genetic distance and phylogenetic analysis, Pennsylvania State University, PA, 1993.

[22] Qin G.Q., Chang H., Cheng G.H., Li J.F., RAPD and RFLP markers of Tibetan goat, J. Northwest Agr. Univ. 1 (1998) 17-20.

[23] Raymond M., Rousset F., GENEPOP version 1.2: Population genetics software for exact tests and ecumenicism, J. Hered. 86 (1995) 248-249.

[24] $\mathrm{SAS}^{\circledR}$ Institute, SAS/STAT Software release 6.1, SAS ${ }^{\circledR}$ Institute, Inc., Cary, NC, 1996.

[25] Saitbekova N., Gaillard C., Obexer-Ruff G., Dolf G., Genetic diversity in Swiss goat breeds based on microsatellite analysis, Anim. Genet. 30 (1999) 36-41. 
[26] Sun J.M., The origin and evolution of goat, J. Chin. Sheep Goat Farming 1 (1997) 6-8.

[27] Takezaki N., Nei M., Genetic distances and reconstruction of phylogenetic trees from microsatellite DNA, Genetics 144 (1996) 389-399.

[28] Tu Y., Sheep and Goat Breeds in China, Shanghai Scientific \& Technical Publishers, Shanghai (P.R. China), 1989.

[29] Tuñón M.J., Gonzale Z.P., Vallejo M., Genetic relationships between fourteen native Spanish breeds of goat, Anim. Genet. 20 (1989) 205-212.

[30] Wang J., Wang Y., Ou Y.X., A study on breeding characters of Tibetan goat, J. Southwest Nationalities Coll., Nat. Sci. Ed. 3 (1995) 272-282 (in Chinese).

[31] Wang J., Wang Y., Ou Y.X., The study of Tibetan goat, J. Sichuan Grassland, 1 (1994) 39-44 (in Chinese).

[32] Wimmers K., Ponsuksili S., Hardgeg T., Valle-Zarate A., Mathur P., Horst P., Genetic distinctness of African, Asian and south American local chickens, Anim. Genet. 31 (2000) 159-165.

[33] Yang J.D., Jian C.S., Wei H., RAPD analysis to $W u$ goats and Small-xiang goats, Hereditas (Beijing), 23 (2001) 521-525.

[34] Yang L., Zhao S.H., Li K., Peng Z.Z., Montgomery G.W., Determination of relationship among five indigenous Chinese goat breeds with six microsatellite markers, Anim. Genet. 30 (1999) 452-456.

[35] Zeuner F.E., A history of domesticated animals, Hutchinson, London, 1963.

To access this journal online: www.edpsciences.org 